\title{
PRACTICE OF PROPER HANDWASHING AND ITS ASSOCIATED FACTORS AMONG STAFF IN A MALAYSIAN UNIVERSITY
}

\author{
San San $\mathrm{Oo}^{1}$, Mohd Salami Ibrahim ${ }^{2 *}$, Myat Moe Thwe Aung${ }^{1}$, Yuzana Mohd Yusop ${ }^{1}$, Azmi Hassan ${ }^{1}$, Nyi Nyi \\ Naing ${ }^{1}$, Rahmah Mohd Amin ${ }^{1}$, Aniza Abd Aziz ${ }^{1}$ and Nik Nor Ronaidi Nik Mahdi ${ }^{1}$

\begin{abstract}
${ }^{1}$ Department of Community Medicine, Faculty of Medicine, Universiti Sultan ZainalAbidin, Jalan Sultan Mahmud, 20400, Kuala Terengganu, Terengganu, Malaysia,

${ }^{2}$ Medical Education Unit, Faculty of Medicine, Universiti Sultan ZainalAbidin, Jalan Sultan Mahmud, 20400, Kuala Terengganu, Terengganu, Malaysia.
\end{abstract}

*Corresponding author: Mohd Salami Ibrahim

Email address: salamiibrahim@unisza.edu.my

\begin{abstract}
In higher education setting throughout the world, the practise of handwashing among staff is essential, particularly in the period of COVID-19 pandemic. Despite the overwhelming evidence on the effectiveness of proper handwashing, the knowledge, attitude and practice of handwashing among the staff of higher education have not been well investigated. Consequently, a cross-sectional study among staff in a university in Malaysia was conducted to identify the prevalence of proper handwashing and its associated factors. The data was collected using questionnaires in Google form from 18th June to 21st June 2020. Statistical analyses involved simple and multiple logistic regression with backward likelihood ratio method. Out of 349 participants, $31.8 \%(95 \% \mathrm{Cl}: 26.9,36.7)$ had proper handwashing practice. The results of the multivariable analysis revealed that the total knowledge scores (OR: 1.04, 95\% Cl: 1.001, 1.071) and the total attitude scores (OR: $1.23,95 \% \mathrm{Cl}: 1.152,1.321)$ were found to be significantly associated with proper handwashing practice among staff. Our study suggests that having the right knowledge and favourable attitudes are crucial towards achieving the proper handwashing practise. Additional supports for promoting a conducive ecosystem may also likely be needed to sustain a long-term practice of proper handwashing.
\end{abstract}

Keywords: knowledge, attitude, practice, handwashing, Malaysia, associated factors, Terengganu

\section{INTRODUCTION}

In the era post coronavirus disease 2019 (COVID19), handwashing is championed as one of the leading public health measures to contain the spread of the pandemic. ${ }^{1}$ Handwashing is recommended in almost every physical contact in public areas. Duration of handwashing varies depending on the loads and circumstances, although 10 to 15 seconds were deemed typically appropriate to eliminate transient flora from the hands. ${ }^{2}$

Many infectious diseases are transmissible via contaminated hands. Diseases causing symptoms of diarrhoea and pneumonia have been shown to be associated with poor hand hygiene especially after certain situations such as using the toilet, coughing or blowing the nose, handling garbage and touching unclean surfaces. ${ }^{3}$ A 2017 report from the United Nations Children's Fund (UNICEF) revealed that illnesses related to both diarrhoea and pneumonia, which can effectively be controlled by handwashing, were responsible for almost $20 \%$ of global mortality among children under the age of five. ${ }^{4}$ Supporting this report is an earlier review of the evidence conducted by Freeman et al in 2014 whose study involved searching, screening, selecting, and appraising 42 studies from 1970 to 2013 on the effectiveness of handwashing. ${ }^{5}$ Their meta-regression analyses established that handwashing after contact with excreta is associated with up to $40 \%$ reduced risk of developing diarrhoeal disease. ${ }^{5}$ Likewise, in the setting of preventing outbreaks caused by viruses, handwashing had been advocated as one of the primary measures to control the spread of the deadly Ebola virus disease. ${ }^{4}$

Thus, extensive studies had been conducted with regards to the practice of proper handwashing and its significant individual and public health benefits. However, to the best of our knowledge, research on proper handwashing among the university staff have not been well investigated. We believe a study among this population is paramount because of the nature of their work which typically involves sitting in a confined office but also having regular contact with a large crowd of people in almost a daily basis. Hence, university staff are at high risk of contracting infectious diseases, including COVID-19, which are transmissible through physical contact. ${ }^{6}$ Consequently, given the gravity of the pandemic, the importance of handwashing as one of the primary measures against the spread of infectious diseases has never been more emphasised.

Consequently, the Ministry of Health of Malaysia $(\mathrm{MOH})$ has launched strong campaigns urging employees to maintain good personal hygiene at the workplace by practising frequent handwashing either with soap or water. ${ }^{7}$ The call was backed by an evidence from Zivich et. al (2018) whose 
systematic review was appraising eleven studies on the effectiveness of handwashing interventions on preventing infectious diseases among employees in office-based workplaces - eight of the eleven were experimental design. ${ }^{8}$ The review which represents a shortcut literature evidence of more than 50 years until 2016 showed that minimal hand-hygiene interventions correspond to a reduction in the incidence of illness among employees. ${ }^{8}$ Further augmenting the call from $\mathrm{MOH}$ is the concern on the easy spread of COVID19 disease since currently there is no effective vaccine and low herd immunity among the Malaysian population. The pressures from the economy and educational sectors have also been magnifying the demand to achieve compliance on proper handwashing practice. Since July 2020, Malaysian higher education had gradually opened itself to the on-campus learning where the influx of students returning to the university is expected to begin from October 2020.

Therefore, this study is conducted to address the crucial gaps in the current educational and public health practices. Based on the findings of participants' knowledge, attitude and practice (KAP) this study aims to provide a firm direction for the next course of interventions and public health strategies for safe higher educational practices in the setting of ongoing global health crisis resulted from the COVID-19 pandemic.

\section{METHODOLOGY}

A cross-sectional study was conducted among Universiti Sultan Zainal Abidin (UniSZA) staff in Gong Badak Campus, Kuala Nerus, from $3^{\text {rd }}$ May 2020 to $21^{\text {st }}$ May 2020 and from $14^{\text {th }}$ June 2020 to

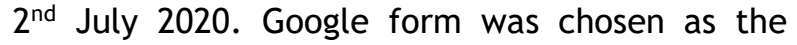
platform for the online questionnaire because of its industry-grade security ${ }^{9}$ and familiarity among potential participants. All UniSZA academic and administrative staff who were able to read and understand Malay language were invited. Those with limited digital literacy or those with restricted access to the internet at the time of recruitment were excluded from this study.

Data were collected using the pre-tested questionnaire. The items in the questionnaire were made up mostly from the validated questionnaires of the previous studies. ${ }^{10,11}$ Public health experts were asked to check the contents, and then the first draft of the questionnaire was distributed to four lecturers from UniSZA Medical Faculty to check the face validity of the questionnaire. After reshaping the questionnaire, the pre-test study was conducted among altogether 30 office staff and medical lectures from UniSZA Medical Faculty using Google Form to evaluate the construct validity and reliability of the questionnaire. As a result of the pre-test study, the items in the questionnaire were revised and edited based on the respondents' comments and the public health expert opinions.
The finalized questionnaire consisted of four sections. Section A probed the socio-demographic characteristics of the respondents. A total of 52 items in Section B with response options of either "yes", "no" or "not sure" assessed the respondents' knowledge on handwashing. Two marks, one mark, and zero mark were given to the correct, wrong, and "not sure" answers, respectively. In this section, another eight items explored the source information about proper handwashing.

Attitude towards handwashing was assessed via ten items with Likert scale scoring in section $C$. Scores of 1, 2, 3, 4, and 5 were assigned for strongly disagree, disagree, neither agree nor disagree, agree and strongly agree responses, respectively. Statements with negative remarks carried reversed scores. Both Sections B and C, which assessed knowledge and attitude, were analysed as continuous numerical variables.

Section D, in contrast, was composed of 31 items to evaluate the respondents' practice about proper handwashing. Based on the Likert scale format, the questionnaire followed the increasing order of frequency from never, rarely, sometimes, often, usually, and always. All of these five categories were defined as $0,1-2,3-4,5-6,7-8$, and $9-10$ out of 10 times, respectively. Reversed score ratings were applied for each negative statement. The highest total practice score was 152. Having scores of at least $75 \%$ and above in practice questions was considered as having proper handwashing practice as in the study done by Nair et al., 2014. ${ }^{11}$ Else, the responses were categorised as inadequate handwashing practices. The reliability tested in the pre-test study showed that the Cronbach's alpha values for knowledge, attitude and practice domains were 0.632, 0.694 and 0.788 respectively.

The questionnaires were distributed through WhatsApp mobile apps or via university email to the staff in the UniSZA Gong Badak Campus from $18^{\text {th }}$ June to $21^{\text {st }}$ June 2020 . Details of the study, confidentiality, and voluntary participation were described together with the questionnaire in Google form. Consent to participate in the survey was taken from all eligible respondents before answering the questionnaire. The information provided was kept private and confidential. To conduct the study among UniSZA staff, the ethics approval was obtained from UniSZA Human Research Ethics Committee (UHREC) on $14^{\text {th }}$ June 2020 (Reference no: UniSZA.C/2/UHREC/628-2 Jld 2 (77)).

The total required sample size for this study was calculated using two proportions formula by the use of power and sample size calculation software. ${ }^{12}$ Based on the pre-test study, the proportion of having proper handwashing among females was found to be $72.7 \%$ while the estimated proportion among males was $57 \%$. The power of the study was set at $80 \%$, and the ratio 
of female to male respondents was taken 1:1. The calculated result showed the required sample size was 144 per group. After considering 20\% expected missing values, data were required to collect from the total of 346 respondents for the study.

Statistical analysis was performed using statistical package for Statistical Package for the Social Sciences (SPSS) version 22. ${ }^{13}$ Data we explored and cleaned. Frequency (percentage) and mean (standard deviation) represented descriptive analyses of categorical data and numerical data, respectively. The prevalence of staff having proper handwashing practice was presented with percentage and its $95 \%$ confidence interval $(\mathrm{Cl})$.

Simple logistic regression was applied to determine the potential candidate significant variables. Multiple logistic regression with backward likelihood ratio method was applied to identify the associated factors of the practice of proper handwashing among UniSZA staff. Multicollinearity and interactions among independent variables were checked. The fit of the final model was assessed by HosmerLemenshow goodness-of-fit test, overall correctly classified percentage from the classification table and the area under the receiver operating characteristic (ROC) curve. Final results were presented by using crude and adjusted odds ratio with $95 \% \mathrm{Cl}$, Wald statistic and corresponding Pvalue. The level of significance (a) was set at less than 0.250 for univariable analysis and less than 0.05 for multivariate analysis.

\section{RESULTS}

Demographic characteristics of respondents

A total of 349 eligible respondents participated in this study. Table 1 showed the details about the socio-demographic characteristics of the respondents. The majority were Malay $(95.1 \%)$, married $(73.6 \%)$, and female $(63.9 \%)$ with a mean age of 38.75 years old. More than half of the respondents were UniSZA academic staff (58.2\%) and had a master or PhD (57.3\%). They had 9.27 years of service on average, and most of the respondents $(61.0 \%)$ received a monthly income of RM 4000 and above.

\section{Prevalence of UniSZA staff having proper handwashing practice}

Out of the total 349 participants, $31.8 \%$ (95\% Cl: $26.9,36.7)$ of them had proper handwashing practice.

\section{Knowledge about proper handwashing among UniSZA staff}

The majority of the respondents (73.9\%) claimed that they knew how to wash their hands properly. In comparison, respondents knew that proper handwashing meant washing their hands with water and soap (94.6\%), or anti-septic hand rub (53.0\%) or alcohol-based hand rub (53.3\%). A much smaller proportion erroneously assumed proper handwashing could be achieved with water only $(24.1 \%)$, wet tissue $(20.3 \%)$, dry tissue $(21.2 \%)$, or hand lotion (10.0\%).

Table 1 Socio-demographic characteristics of respondents $(n=349)$

\begin{tabular}{llcc}
\hline Variables & & Mean (SD) & Frequency (\%) \\
\hline Age & in years & $38.75(8.67)$ & \\
Gender & Male & & $126(36.1)$ \\
& Female & & $223(63.9)$ \\
Ethnicity & Malay & $332(95.1)$ \\
& Non-Malay & $17(4.9)$ \\
Occupation & Academic staff & $203(58.2)$ \\
& Administrative staff & $146(41.8)$ \\
Educational level & Attaining up to Degree & & $149(42.7)$ \\
Year of service & Having master or PhD & & $200(57.3)$ \\
Monthly household income & In years & $9.27(6.94)$ & $136(39.0)$ \\
& <RM4000 & & $213(61.0)$ \\
Marital status & RM4000 & & $92(26.4)$ \\
& Single & & $257(73.6)$ \\
\hline
\end{tabular}

Related to the number of steps in proper handwashing, more than half of the respondents replied that they knew about it $(53.9 \%)$, could identify the correct number of steps (53.0\%), and knew that they should wash their hands for more than 20 seconds (55.6\%). On the subject of conditions to wash hands, the majority of the respondents recognised when they should wash their hands. They knew that they had to wash hands before preparing foods $(88.8 \%)$, after preparing foods $(84.5 \%)$, before eating $(93.1 \%)$, and after eating (90.8\%). Furthermore, they also knew that they should wash their hands in the following conditions: before $(53.6 \%)$, and after using toilets $(92.0 \%)$, after blowing $(82.2 \%)$ or holding their nose $(64.8 \%)$, after coughing $(73.1 \%)$, 
after touching non-living things $(61.6 \%)$ or animals (77.7\%), after using public transportation $(72.8 \%)$, after coming back from outside (73.9\%), after shaking hands with others $(69.1 \%)$, when hands look dirty (84.0\%), when hands smell bad (91.4\%) and after hands are soiled (86.2\%).

Concerning the commonly neglected parts of hands, while washing, most of the respondents could recognise these places; thumb (49.3\%), fingertips (57.3\%), nails (71.3\%), dorsum of hands (55.9\%), and web spaces (60.5\%). Additionally, they had the knowledge that disease can be transmitted via unclean/dirty hands (90.5\%). They knew that the bacteria and infection could stay in hand jewellery (88.3\%), long nails (94.0\%), watches $(75.6 \%)$, and cracks in hands $(78.2 \%)$. They could identity unclean hands can cause diseases such as respiratory tract infections (72.2\%), skin infections $(81.1 \%)$, cholera $(68.8 \%)$, food poisoning $(81.1 \%)$, hand, foot, and mouth disease (75.9\%), typhoid fever (53.9\%), Covid-19 (84.0\%) and Hepatitis A (46.7\%). Information on handwashing was mostly retrieved from the internet $(84.8 \%)$, mass media $(71.1 \%)$, campaign (67.6\%), and school (61.9\%). Meanwhile, friends (37.5\%), seminar (40.1\%), newspaper (43.8\%), and home $(44.7 \%)$ made up the remaining source of information.

3.4.

\section{Attitude towards handwashing among UniSZA staff}

The majority of the respondents agreed that handwashing could reduce the risk of contracting diseases (90.2\%) and prevent the spread of infection to others $(88.2 \%)$. They concurred that hand hygiene is an essential issue (84.2\%) and should become a routine practice $(80.8 \%)$. Moreover, most of them assumed that following handwashing steps was easy $(75.7 \%)$. They felt guilty when they omit to handwash $(72.8 \%)$, and they felt frustrated when others neglected their handwashing (75.9\%).

Meanwhile, some respondents had an attitude that handwashing was time consuming (25.2\%), and the frequency of handwashing makes it difficult for them to carry it out as often as necessary $(28.9 \%)$. Considering the facilities available for hand hygiene in their faculty, the majority of subjects $(71.1 \%)$ were satisfied with them.

\section{Practice of proper handwashing among UniSZA staff}

While washing, most of the respondents always use water and soap (48.4\%), anti-septic hand rub $(18.6 \%)$, or alcohol swab (13.5\%). However, some participants washed their hands with water only (46.1\%), damp/wet tissue (12.3\%), dry tissue (16.9\%), or hand lotion $(6.9 \%)$. About $56.2 \%$ of the respondents could answer the proper steps in handwashing correctly.

Out of 349 respondents, $23.8 \%$ claimed that they always washed their hands for more than 20 seconds. After handwashing, the respondents always did dry their hands using an automatic hand dryer $(13.8 \%)$ or dry tissue (disposable) (37.2\%) while some dried their hands with towels or handkerchief $(30.1 \%)$ or let the hands dry naturally $(26.9 \%)$.

The practice of handwashing was noticeably less frequent in some situations. These include before using toilets $(23.2 \%)$, after blowing the nose (43.6\%), after holding nose $(28.1 \%)$, after coughing $(31.5 \%)$, after touching non-living things (24.6\%), after touching animals (44.7\%), after going out from outside (38.7\%), after using public transportation $(38.4 \%)$ and after shaking hands with others (31.8\%). On the other hand, more than half of the respondents claimed they did wash their hands before $(55.3 \%)$ and after preparing meals $(57.3 \%)$, before $(70.5 \%)$ and after having meals $(65.3 \%)$, after using toilet $(63.9 \%)$, when hands look dirty $(58.5 \%)$, when hands get foul smell bad $(73.6 \%)$ and after hands were soiled (61.6\%).

Total knowledge and attitude scores towards handwashing

The mean (S.D.) score for the knowledge of handwashing was 85.6 (13.6), with minimum and maximum scores of 12 and 102.00 , respectively. Meanwhile, the mean score for the attitude towards handwashing was 40.57 (7.65), with minimum and maximum scores of 18 and 50.00, respectively.

\section{Associated factors of the practice of proper handwashing among UniSZA staff}

Univariable analysis using simple logistic regression revealed the significant factors associated with the practice of proper handwashing among the respondents were occupation (unadjusted OR: $0.56,95 \% \mathrm{Cl}: 0.348$, $0.896 ; \mathrm{p}=0.016$ ), educational levels (unadjusted OR: $2.00,95 \% \mathrm{Cl}: 1.242,3.208 ; \mathrm{p}=0.004)$, total knowledge scores (unadjusted OR: $1.08,95 \% \mathrm{Cl}$ : $1.048,1.108 ; p<0.001)$, and the total attitude scores towards handwashing (unadjusted OR: 1.25, 95\% Cl: 1.175, 1.338; $\mathrm{p}<0.001$ ) (Table 2).

After controlling confounders using multiple logistic regression, the results revealed that total knowledge scores (adjusted OR: 1.04, 95\% Cl: $1.001,1.071 ; p=0.046)$ and the total attitude scores towards handwashing (adjusted OR: 1.23, $95 \% \mathrm{Cl}: 1.152,1.321 ; \mathrm{p}<0.001$ ) were found to be the significant associated factors of proper handwashing practice (Table 2 ).

There were no interaction and multicollinearity problems detected. Hosmer-Lemenshow test $(p=$ 0.061 ), overall correctly classified percentage (73.9\%) and area under ROC curve (77.9\%; $95 \% \mathrm{Cl}$ : $73.1,82.8 ; p<0.001)$ were applied to check the fit of the statistical model. The results of the final model indicated that additional one-mark increase in knowledge scores corresponded with $4 \%$ increased probability in practising proper 
handwashing. Likewise, a 23\% increase in the likelihood of practising proper handwashing was seen with an additional one-unit increase in attitude scores (Table 2).

\section{DISCUSSION}

Handwashing is one of the main pillars of public health measures to break the spread of many infectious diseases, including COVID-19. Our study demonstrated the majority of respondents wield an excellent level of knowledge on handwashing. In comparison, almost one-third were practising proper handwashing. These results are a significant departure from the findings of previous studies on the knowledge and practice of handwashing. For example, a 2013 study by Rabbi \& Dey ${ }^{14}$ involving more than thirty thousand Bangladeshi populations from 50 sub-districts revealed limited knowledge and practice of handwashing, where only $8 \%$ of the respondents were reporting washing their hands with soap. Similarly, in a study among 523 undergraduate medical students in India, Modi et al. $(2017)^{15}$ reported that only $12.2 \%$ of respondents demonstrated good knowledge of hand hygiene. In a slightly more heterogenous but smaller population sample of 144 medical and nursing students, Nair et al. $(2014)^{16}$ reported the prevalence of respondents with good knowledge of hand hygiene was only at 9\%. All of these studies were conducted in developing countries and to a certain degree, comparable to our population. However, they were conducted before the COVID-19, suggesting differences in findings may reflect the significant change in public health practices following the pandemic.

The massive public health promotions likely drive this change. Our study was conducted in May 2020, two months after the initiation of Movement Control Order (MCO), the Malaysian version of multiple stages of lockdown policy. MCO, together with the other campaigns on physical distancing, handwashing, and face mask covering, constitute a broader strategy to curb the local spread of COVID-19. The scale of these campaigns was unprecedented, matching the magnitude of the public health crisis brought upon by the pandemic. Supporting this assertion is our results which revealed the internet, campaigns, and mass media were rated as the top three sources of information on handwashing. The outcome is not surprising; large-scale health promotion has been shown to improve the knowledge, attitude and practice of handwashing. In 2013, Rabbi \& Dey ${ }^{14}$ reported that national-based project called water, sanitation, and hygiene (WASH) health campaign programs resulted in improved knowledge and practice of hand hygiene among a population in India from $8 \%$ to $22 \%$.

The change in knowledge, attitude, and practice caused by the COVID-19 pandemic may also extend beyond just merely observing the laws and policies. The pandemic has brought a fundamental shift in almost every aspect of human life. In Malaysia, all religious sites such as mosques, churches, and temples were initially closed for several months before being allowed to open with strict standard operating procedures (SOPs). The closure of religious sites was a significant mark of social impact on a multi-cultural society whose association with religious practices are rich and strong. New SOPs were also being introduced for shops, supermarkets, libraries, and almost on all sites wherever observing physical distancing is warranted. Together with these SOPs, education on the importance of hand sanitising and handwashing is promoted so the general public will understand and support these SOPs as well as becoming agents of change to remind its members to embrace the new norms. An insightful report published by the International Water Association (IWA) in June 2020 demonstrated that more than $60 \%$ increase in the proper practice of handwashing among the residents of northern states of India compared with the period before COVID-19. ${ }^{17}$ Consistent evidence was also revealed in the field of business and economies. Consumer behaviour from the United States of America (U.S.) has changed significantly post COVID-19, where concerned over the public health among $85 \%$ of respondents had manifested in their increased expenditure towards healthrelated goods and services. ${ }^{18}$ Although the consumer behaviour in the U.S. population may traditionally be different than the Malaysian population due to differences in socio-economic profiles, health concerns as the primary driver of change in consumer behaviour are likely universal to all world's population. Hence, these observations provide further insight into the significant shift in cultures within the community, which has a direct impact on knowledge, attitude, and practice of handwashing. 
Table 2: Factors associated with proper handwashing practice among UniSZA staff

\begin{tabular}{|c|c|c|c|c|c|c|c|c|c|c|}
\hline \multirow{2}{*}{ Variables } & \multicolumn{2}{|c|}{ Mean (SD) } & \multicolumn{4}{|c|}{ Simple Logistic Regression } & \multicolumn{4}{|c|}{ Multiple Logistic Regression } \\
\hline & $\begin{array}{l}\text { Having } \\
\text { practice }\end{array}$ & $\begin{array}{l}\text { Not having } \\
\text { practice }\end{array}$ & B & $\begin{array}{c}\text { Crude OR } \\
(95 \% \mathrm{Cl})\end{array}$ & $\begin{array}{c}\text { Wald } \\
\text { Statistic }\end{array}$ & $\begin{array}{c}P \\
\text { Value }\end{array}$ & B & $\begin{array}{l}\text { Adjusted OR } \\
\quad(95 \% \mathrm{Cl})\end{array}$ & $\begin{array}{c}\text { Wald } \\
\text { Statistic }\end{array}$ & $\begin{array}{c}P \\
\text { Value }\end{array}$ \\
\hline $\begin{array}{l}\text { Knowledge } \\
\text { scores }\end{array}$ & $91.33(8.51)$ & $82.93(14.08)$ & 0.07 & $1.08(1.048,1.108)$ & 27.15 & $<0.001$ & 0.03 & $1.04(1.001,1.071)$ & 4.00 & 0.046 \\
\hline $\begin{array}{l}\text { Attitude } \\
\text { scores }\end{array}$ & $45.23(3.50)$ & $38.39(8.08)$ & 0.23 & $1.25(1.175,1.338)$ & 46.74 & $<0.001$ & 0.21 & $1.23(1.152,1.321)$ & 36.14 & $<0.001$ \\
\hline
\end{tabular}

a Backward likelihood Multiple Logistic regression; There were no interaction and multicollinearity problems detected.

The model reasonably fits well.

Hosmer-Lemenshow test $(p=0.061)$,

overall correctly classified percentage (73.9\%) and the area under ROC curve $(77.9 \%, 95 \%$ CI: $73.1,82.8 ; p<0.001)$ were applied to check fit of the model.

Consequently, our study proves the improved knowledge and attitude were significantly associated with the practice of proper handwashing. With the fitness of the statistical model is well-substantiated, our study shows that a university staff who scores one unit higher in the knowledge score is associated with $4 \%$ more likely to practice proper handwashing. Likewise, $23 \%$ increased in the practice of proper handwashing is demonstrated among university staff whose favourable attitude towards handwashing improved by one unit of scoring. These meaningful results vindicate the primary premise that education, rather than law, is the central public health strategy where the right knowledge and the right attitude is a sine-qua-non for the right practice. The intrinsic association between knowledge, attitude and practice of handwashing, therefore, serve as a framework for public health strategy to improve the proper practice of handwashing in the long term.
Nonetheless, since there was a considerable gap to achieve majority practice of proper handwashing despite excellent knowledge and favourable attitude among most of the respondents, our study also suggests that there are other contributing factors influencing the practice of proper handwashing. These factors may include ease of access. For example, the availability of public access to a water tap with sinks and soap may limit the frequency of practising proper handwashing. Likewise, the financial aspect may also need to be considered to ensure sustainable access. These factors are particularly important to be considered so the facilities and resource allocations can be tailored to match the needs of the new norms. 
There are some limitations to our study. Firstly, since the era of COVID-19, proper handwashing has become a much more sensitive issue. Lack of proper handwashing may invite greater scrutiny. Hence, we neither asked nor performed analyses based on faculties or departments, which put a limit for more targeted analyses and discussions. Nonetheless, this is warranted to observe the ethical practice of protecting research participants against any element of potential vulnerability. Secondly, our study was conducted at the time when Malaysia was gradually relaxing its restrictions and slowly progressing towards a recovery period. Businesses were allowed to open, and local travels had been permitted. The evolution of COVID-19 prevalence and the degree of policy restriction may put a limit on the generalisation of our findings. For example, it is unclear if the gaps between the proper practice of handwashing with knowledge and attitude will be similar if this study were conducted during the initial period of MCO, when policies and regulations were much stricter.

Finally, the cross-sectional design of our study enables access to a broad sample of the population and generate meaningful differences compared with studies from the pre-COVID era. To further consolidate the findings of this study, qualitative studies to explore the change of knowledge, attitude, and behaviour on handwashing will likely to prove useful. Qualitative studies may provide a deeper understanding and insight into the barriers and facilitators of the proper practice of handwashing. Consequently, this may illuminate many aspects of the results of this study, so more meaningful public health intervention can be formulated either at the institutional or national level.

\section{CONCLUSIONS}

In summary, our study demonstrated that the majority of the staff of a higher educational institute in Malaysia wield an excellent level of knowledge and right attitude on handwashing. This translates into proper practice of handwashing in almost a third of all respondents of this study. Concentrated efforts on public health promotions and a fundamental change in culture and behaviour towards health-related issues following the COVID-19 pandemic likely explain these findings. However, the practice of proper handwashing is not exclusively dictated by the right knowledge and the right attitude. More efforts and strategies need to be invested in illuminating and addressing barriers of proper practice of handwashing. Future research may include qualitative studies to attain an in-depth understanding of all factors influencing the proper practice of handwashing.

\section{ACKNOWLEDGMENTS}

We would like to extend our great appreciation to all staff of UniSZA Gong Badak campus, Year 3
MBBS students (2019-2020) and all research participants. The conduct of this research was made possible by their administrative, logistics and technical supports as well as voluntary participation.

\section{CONFLICTS OF INTEREST}

The authors declare no conflict of interest.

\section{FUNDING}

This research received no external funding.

\section{REFERENCES}

1. World Health Organization (WHO), Promote hand hygiene to save lives and combat COVID-19. 2020.2 (Online). https://www. who.int/southeastasia/news /detail/04-05-2020-promote-hand-hygieneto-save-lives-and-combat-covid- 19. (accessed 29 July 2020).

2. Jemal S. Knowledge and Practices of Hand Washing among Health Professionals in Dubti Referral Hospital, Dubti, Afar, Northeast Ethiopia. Adv Prev Med 2018;1-7.

3. Dajaan DS, Addo HO, Ojo L, Amegah KE, Loveland F, Bechala BD, et al. Hand washing knowledge and practices among public primary schools in the Kintampo Municipality of Ghana. Int J Community Med Public Heal 2018; 5(6):2205.

4. The Global Handwashing Partnership. 2017. https: / /globalhandwashing.org/abouthandwashing/why-

handwashing/health/\#_edn4 (accessed 25 September 2020).

5. Freeman $M C$, Stocks $M E$, Cumming $O$, Jeandron A, Higgins JPT, Wolf $J$, et al. Hygiene and health: systematic review of handwashing practices worldwide and update of health effects. Trop Med Int Health 2014; 19(8):906-16.

6. Shereen MA, Khan S, Kazmi A, Bashir N, Siddique R. COVID-19 infection: Origin, transmission, and characteristics of human coronaviruses. Journal of Advanced Research 2020; 24:91-8.

7. Malaysia, Ministry of Health $(\mathrm{MOH})$. Covid19: Management Guidelines for Workplaces. 2020. http://covid-19.moh.gov.my/garispanduan/garis-panduan-

$\mathrm{kkm} /$ Annex_25_COVID_guide_for_workplac es_22032020.pdf (accessed 9 September 2020).

8. Zivich PN, Gancz AS, Aiello AE. Effect of hand hygiene on infectious diseases in the office workplace: A systematic review. Am $\mathrm{J}$ Infect Control 2018; 46(4):448-55. 
9. Google. Google Privacy Policy. 2019. Available online: https://policies.google.com/privacy (accessed on 16-August-2020).

10. Ergin A, Bostanci M, Önal Ö, Bozkurt Al, Ergin N. Evaluation of students' social hand washing knowledge, practices, and skills in a university setting. Cent Eur J Public Health 2011; 19(4):222-7.

11. Nawab T, Mehnaz S, Jafar Abedi A, Rehman Safwi S, Khalique N, Athar Ansari M, et al. Knowledge Attitude and Practice of hand hygiene among medical and nursing students in a tertiary teaching hospital. ljsar 2015; 2(6):29-39.

12. Dupont, WD. and Plummer, WD. Power and Sample Size Calculations: A Review and Computer Program. Controlled Clinical Trials 1990; 11:116-128.

13. IBM Corp. Released 2013. IBM SPSS Statistics for Windows, Version 22.0. Armonk, NY: IBM Corp.

14. Rabbi SE, Dey NC. Exploring the gap between hand washing knowledge and practices in Bangladesh: a cross-sectional comparative study. BMC Public Health 2013;13(1):89.

15. Modi PD, Kumar P, Solanki R, Modi J, Chandramani S, Gill N. Hand Hygiene Practices Among Indian Medical Undergraduates: A Questionnaire-Based Survey. Cureus 2017; 9(7):e1463.

16. Nair SS, Hanumantappa R, Hiremath SG, Siraj MA, Raghunath P. Knowledge, Attitude, and Practice of Hand Hygiene among Medical and Nursing Students at a Tertiary Health Care Centre in Raichur, India. ISRN Prev Med 2014; 7:1-4.

17. International Water Association. COVID-19 leading to behavioural change in handwashing? 2020. https://iwanetwork.org/covid-19-leading-tobehavioural-change-in-handwashing/ (accessed 21 Aug 2020).

18. Accenture. COVID-19 Will Permanently Change Consumer Behavior. 2020. https://www.accenture.com/usen/insights/consumer-goodsservices/coronavirus-consumer-behaviorresearch (accessed 7 September 2020) 